\title{
Analysis of Scaling-Up Resistances from Leaf to Canopy Using Numerical Simulations
}

\author{
Adriana C. Furon, Jon S. Warland,* and Claudia Wagner-Riddle
}

\begin{abstract}
A multi-layer model, combining Lagrangian dispersion at the canopy level with Ohm's Law analogy at the leaf level, was used in numerical simulations to assess the leaf-to-canopy scale translation of surface resistances. The model produced unique profiles of fluxes and scalar concentrations that satisfied both the dispersion and leaf models. Environmental factors and canopy architecture were varied, and stomatal conductance was simulated using either a simple relationship with net radiation or the Ball and Berry model to account for feedback mechanisms. Results showed that, when the assumptions of the PenmanMonteith equation were met, scaled-up leaf conductance closely matched the bulk canopy conductance. However, as the scenarios modeled departed from the ideal conditions of Penman-Monteith, the agreement decreased. In particular, correct estimation of the aerodynamic resistance, through correct parameterization of the roughness length for sensible heat, was identified as a key issue.
\end{abstract}

$\mathrm{O}$ NE OF THE MOST WIDELY USED APPROACHES to modeling or estimating evapotranspiration is the PenmanMonteith equation (Monteith, 1965); this "combination equation" includes the effects of both the energy supply and the dispersion of water vapor away from the evaporating surface and it has been applied at both the leaf level and, more often, at the canopy level. The attractiveness of its application at the whole canopy level is that only a few parameters are needed; however, "correctness and usefulness of this equation depends entirely on how accurately and easily we can determine the bulk aerodynamic and surface [canopy] resistances" (Raupach and Finnigan, 1988).

In the original formulation of the combination equation (Monteith, 1965), it was assumed that the bulk canopy resistance $\left(r_{\mathrm{c}}\right)$ was primarily dependent on stomatal resistance. Later on, however, Monteith (1981) recognized that "it is not evident a priori whether $r_{\mathrm{c}}$ can be regarded as a physiological resistance depending mainly on stomatal components or whether it contains a significant aerodynamic element"; at the same time, he cited a number of theoretical and experimental studies whose results supported the physiological significance of the bulk canopy resistance. The appeal of a strong physiological component in the bulk canopy resistance is that it justifies the leaf-to-canopy scale translation of resistances or, more precisely, of conductances. The scaled-up stomatal resistance is thus defined by $R_{\mathrm{tot}}=\left[\int_{0}^{h} g_{\mathrm{st}} \Lambda d z\right]^{-1}$, where $h$ is canopy height,

Department of Land Resource Science, Univ. of Guelph, Guelph, ON, Canada N1G 2W1. Received 25 Nov. 2006. *Corresponding author (jwarland@uoguelph.ca).

Published in Agron. J. 99:1483-1491 (2007). Modeling

doi:10.2134/agronj2006.0335

(c) American Society of Agronomy

677 S. Segoe Rd., Madison, WI 53711 USA $g_{\text {st }}$ is stomatal conductance, and $\Lambda$ is the leaf area density. If $r_{\mathrm{c}}$ is purely physiological, then $r_{\mathrm{c}}=R_{\text {tot }}$.

The validity of scaling-up from stomatal to canopy resistance has not always been supported by empirical studies. Rochette et al. (1991) measured leaf conductance within a maize canopy and concluded that none of the scaling-up methods they tried were appropriate to estimate the bulk canopy resistance calculated as a residual term in the Penman-Monteith equation $\left(r_{\text {res }}\right)$. This paper distinguishes between empirical estimates of canopy resistance $\left(r_{\text {res }}\right)$ calculated assuming the other terms in Penman-Monteith contain no systematic errors and the "true" canopy resistance $\left(r_{\mathrm{c}}\right)$, which may or may not be purely physiological. In a careful examination of the problem, Raupach (1995) analytically compared different model canopies and concluded that, for typical dry canopies, $r_{\text {res }}$ was close to the inverse of the parallel sum of leaf conductances and is, therefore, approximately a physiological parameter of the system. On the other hand, Alves et al. (1998) stated that "the bulk surface [canopy] resistance of dense crops cannot be obtained by simple averaging of stomatal conductances because the driving force [profile of saturation deficit] is not kept constant within the canopy."

Different approaches have been used to address the problem of scaling-up from leaf to canopy: direct measurements of leaf stomatal resistance at different levels in the canopy (Rochette et al., 1991), analysis of the behavior of (observed) $r_{\text {res }}$ (Alves et al., 1998), analytical integration of a multi-layer canopy model to a single-layer model (Shuttleworth, 1976; Raupach, 1995), and simulation of evapotranspiration with a multi-layer canopy where the modeled leaf stomatal resistances were compared to the residual term $r_{\text {res }}$ (Raupach and Finnigan, 1988; Paw U and Meyers, 1989). An underlying assumption in these approaches has been that the Penman-Monteith equation can adequately describe the scenarios under consideration, which may not always be the case. When the Penman-Monteith equation is used to describe situations that depart markedly from the ideal situation for which it was derived (e.g., full canopy cover, aerodynamic resistance for heat and vapor equal, etc.), the physiological significance of the empirical canopy resistance, $r_{\text {res }}$, is expected to be compromised.

It is practically impossible to measure with certainty all the relevant terms under field conditions to empirically analyze the scaling-up problem. Use of a numerical ideal canopy model (ICM), which can simulate both leaf behavior and canopy turbulent dispersion, could provide insight into the circumstances when $r_{\text {res }} \neq R_{\text {tot }}$, if the ICM also correctly simulates $r_{\text {res }}=R_{\text {tot }}$ when the assumptions of Penman-Monteith are strictly met. In

Abbreviations: B\&B, Ball and Berry model; D\&M, Denmead and Millar model; ICM, ideal canopy model; P-M, Penman-Monteith equation. 
the work reported here, an ICM was developed to study the role of in-canopy turbulent dispersion on the scaling-up issue. The model simulates leaf-level function in the canopy layers where stomatal resistance is simulated by either the simple Denmead and Millar (1976) formulation or the more sophisticated Ball and Berry model (Ball et al., 1987). Dispersion in the ICM was simulated using the Lagrangian dispersion analysis of Warland and Thurtell (2000), which describes the relationship between the source strength of scalar in each canopy layer and the resultant concentration profile. This study asks two questions: (i) does P-M describe the behavior of an ideal multi-layer canopy wherein leaf functioning is determined by a physiological model and transport is governed by Lagrangian dispersion? (ii) If so, under what circumstances does P-M fail to reproduce the ICM? Though it is possible that this model may fail for different reasons than empirical measurements, the results will nonetheless provide insight into the problem and a guide for future field work.

This study develops an ICM to investigate the relationship between $r_{\text {res }}$ and $R_{\text {tot }}$ as conditions deviate from the ideal assumptions of Penman-Montieth. The study is heuristic in nature; the ICM is not intended to quantitatively reproduce the 'true' behavior of the system, however, in so far as its output is logical and qualitatively consistent with observed canopy behavior, it can provide insight into how violations of the underlying assumptions lead to discrepancies between $r_{\text {res }}$ and $R_{\mathrm{tot}}$.

In the following sections, we first review the PenmanMonteith equation and the various resistances used in the analysis. Second, we present the ICM used in the study and discuss the model outputs. The final sections discuss the use of the ICM in a variety of ways to diagnose the scaling-up problem.

\section{THE PENMAN-MONTEITH EQUATION}

This section reviews the Penman-Monteith (P-M) equation as a prelude to addressing the scaling-up problem. The P-M equation for evapotranspiration from a surface $\left(\lambda E_{\text {tot }}\right)$ states:

$$
\lambda E_{\text {tot }}=\frac{\Delta A_{\text {tot }}+\left(\rho_{\mathrm{a}} c_{\mathrm{p}} D / r_{\mathrm{a}}\right)}{\Delta+\gamma\left(1+r_{\mathrm{c}} / r_{\mathrm{a}}\right)}
$$

where $\Delta$ is the slope of the saturation vapor pressure against temperature, $\gamma$ is the psychrometric coefficient, $\rho_{\mathrm{a}}$ is air density, $c_{\mathrm{p}}$ is the specific heat of air at constant pressure, $A_{\text {tot }}$ is the total energy available to the system (net radiation less soil heat flux), $r_{\mathrm{a}}$ is the aerodynamic resistance between the source height and the reference height, and $D$ is the vapor pressure deficit at reference height. Note that, because P-M is a "bigleaf" model and does not differentiate between canopy and soil components, the evapotranspiration and available energy are explicitly stated as totals for the surface (i.e., including both canopy and soil).

Several issues with the application of P-M can be identified. First, the essential assumption in P-M is that sources of sensible and latent heat have the same temperature (Monteith, 1981). Therefore, any situation where the source-sink temperatures for sensible and latent heat are different should not be expected to be properly described by this equation. Thom (1975) is more precise when he states that $r_{\mathrm{c}}$ can be an accurate measure of the bulk stomatal resistance of a particular plant community only insofar as the aerodynamic values of vapor pressure and canopy temperature provide good estimates of the actual mean conditions on the surfaces of the transpiring elements. Strict application of this assumption requires that the vertical profile of the Bowen ratio is constant, since otherwise the mean source heights for water vapor and sensible heat will be different, and therefore at different temperatures.

Another significant issue is that the bulk aerodynamic resistance, $r_{\mathrm{a}}$, must be correctly estimated. As an example of the difficulties inherent in this task, Paw U and Meyers (1989) showed, through numerical experiments, that the displacement heights for water vapor flux and heat flux were affected by changes in stomatal resistances. This result suggests that $r_{\mathrm{a}}$ may not be a strictly aerodynamic parameter, but depend in part on stomatal functioning.

Finally, as the canopy cover decreases, the meaning of $r_{\mathrm{c}}$ becomes increasingly unclear since the soil begins to have a greater role within the system. On the one hand, the big-leaf approach of P-M does not distinguish between soil and canopy; on the other hand, when studying the relationship between canopy and leaf resistances, it does not seem justifiable to include the soil contribution to the canopy energy budget. The analysis below examines a full canopy (leaf area index $\Lambda=4$ ) to minimize this issue; further, only the energy available to the canopy and the canopy transpiration are included in the calculation of $r_{\text {res }}$ from the P-M equation.

\section{Resistance Calculations for the Scaling-Up Analysis An Expression for the Residual Resistance}

The following rearrangement of the Penman-Monteith equation was used to calculate the bulk canopy resistance as a residual:

$$
r_{\text {res }}=\left[\frac{\Delta\left(A_{\mathrm{c}}-\lambda E_{\mathrm{c}}\right)}{\lambda E_{\mathrm{c}} \gamma}-1\right] r_{\mathrm{a}}+\frac{\rho_{\mathrm{a}} c_{\mathrm{p}} D}{\lambda E_{\mathrm{c}} \gamma}
$$

where only the canopy energy budget (available energy, $A_{\mathrm{c}}=R_{\mathrm{n}}-G$, and transpiration, $\lambda E_{\mathrm{c}}$ ) is considered to avoid the confounding influence of the soil.

When the first term on the right-hand-side of Eq. [2] is close to zero, the magnitude of $r_{\text {res }}$ becomes independent of the value of the bulk aerodynamic resistance. This condition occurs when:

$$
\frac{\lambda E_{\mathrm{c}}}{A_{\mathrm{c}}}=\frac{\Delta}{\Delta+y}
$$

Under the conditions expressed by Eq. [3], errors in the estimation of $r_{\mathrm{a}}$ have no impact on the calculation of $r_{\text {res }}$. This condition, known as equilibrium evaporation, is utilized in the analysis below to examine the relationship between $r_{\text {res }}$ and $R_{\text {tot }}$ in the ICM independent of $r_{\mathrm{a}}$. 


\section{Scaled-Up Stomatal Resistance}

The total (scaled-up) canopy resistance from the ICM is given by the inverse of the parallel sum of leaf conductances:

$$
R_{\mathrm{tot}}=\left[\sum_{i=1}^{i=n} g_{\mathrm{st} i} \Lambda_{i}\right]^{-1}
$$

where $g_{\mathrm{st} i}$ is the leaf conductance for layer $i, \Lambda_{i}$ is the leaf area for layer $i$, and $n$ is the number of layers into which the canopy has been divided.

\section{Estimation of the Bulk Aerodynamic Resistance}

The following relationships are assumed in the estimation of the bulk aerodynamic resistance:

$$
\begin{gathered}
d=d_{\mathrm{H}}=0.63 h \\
z_{0 \mathrm{M}}=0.13 h
\end{gathered}
$$

where $d$ is the zero-plane displacement height for momentum, $d_{\mathrm{H}}$ is the zero-plane displacement height for heat and water vapor, $h$ is crop height, and $z_{0 \mathrm{M}}$ is the roughness length for momentum. The roughness length for heat and water vapor is parameterized as either $z_{0 \mathrm{H}}=$ $z_{0 \mathrm{M}}$ or $\mathrm{z}_{\mathrm{OH}}=0.2 z_{0 \mathrm{M}}$. Both parameterizations are common in the literature (Choudhury et al., 1987; Raupach and Finnigan, 1988; Rochette et al., 1991; Alves et al., 1998). This issue is discussed in more detail in the Results and Discussion sections.

The bulk aerodynamic resistance is defined by:

$$
r_{\mathrm{a}}=\frac{\ln \left(\frac{x-d}{z_{0 \mathrm{M}}}\right) \ln \left(\frac{x-d_{H}}{z_{0 \mathrm{H}}}\right)}{k^{2} u_{x}}
$$

where $k=0.4$ is von Kármán's constant; $x$ is reference height, and $u_{x}$ is wind speed at reference height. Atmospheric stability is not considered in this study.

Because $r_{\mathrm{a}}$, and therefore $r_{\text {res }}$, can be computed using either parameterization of $z_{0 \mathrm{H}}$, this paper will distinguish $r_{\text {resM }}$ for $z_{0 \mathrm{H}}=z_{0 \mathrm{M}}$ and as $r_{\mathrm{resH}}$ for $z_{0 \mathrm{H}}=0.2 z_{0 \mathrm{M}}$. When the distinction is not relevant to the discussion, the subscript will not be used.

\section{NUMERICAL SIMULATIONS}

A brief description of the two basic components of the ICM and the iterative procedure used to determine canopy source and concentration profiles of water vapor and sensible heat will be provided in the first part of this section. The second part presents the range of conditions used as input to the model.

\section{The Ideal Canopy Model}

The model iteratively solves for flux and concentration profiles of water vapor and temperature (sensible heat), which simultaneously satisfy Ohm's Law analogy at the leaf-level and turbulent dispersion through the canopy. A Lagrangian dispersion model relates concentration and source profiles; these in turn must satisfy the leaf-level model. The iteration procedure finds values of foliar and air temperature and humidity, which satisfy both leaf and canopy models. The gradients of temperature and humidity between the leaf and the air must produce fluxes from Ohm's Law analogy, which, when used in the dispersion equation, produce identical air temperature and humidity profiles. The dispersion matrix couples the model layers and guarantees a unique solution because, for a given set of turbulence conditions, there is one unique concentration profile for a given source profile (Warland and Thurtell, 2000).

\section{The Lagrangian Dispersion Matrix}

The dispersion matrix, described in detail in Warland and Thurtell (2000), relates source and concentration profiles of any scalar within and above the canopy. It allows the calculation of the profile of concentration gradients of some scalar (e.g., temperature) that corresponds to a given spatial distribution of the respective source-sink (e.g., sensible heat flux profile) since the concentration gradient in each layer is defined by the sum of the gradients created by each source layer. Mathematically:

$$
\left.\frac{\partial c}{\partial z}\right|_{i}=\mathbf{M}_{i j} q_{j}
$$

where $i$ and $j$ are the layer at the air level and source level, respectively; $\mathbf{M}_{i j}$ is the dispersion (square) matrix; $q_{j}$ defines the source spatial distribution (i.e., source strength in each canopy layer) and $\left.\frac{\partial c}{\partial z}\right|_{i}$ is the resultant profile of concentration gradients for the scalar under consideration. Translating the profile of concentration gradients into a profile of scalar concentrations only requires that the scalar concentration at one point in the system be known (e.g., temperature at reference height).

The dispersion matrix, $\mathbf{M}_{i j}$, is derived from Lagrangian dispersion theory (Taylor, 1921; Raupach, 1987). According to this theory, from the initial time when the particles are "marked" the particle cloud initially disperses in a linear fashion and at large travel times the cloud becomes diffusive; these are the near-field and far-field regimes, respectively. The average time over which a particle's motion remains correlated with its previous movements is the Lagrangian time scale $\left(\tau_{L}\right)$. The profiles of the turbulence statistics $\tau_{\mathrm{L}}$ and $\sigma_{\mathrm{w}}$ (standard deviation of the vertical wind speed), within and immediately above the canopy are the only inputs needed to define $\mathbf{M}_{i j}$. In the present study, empirical equations, following van den Hurk and McNaughton (1995), were used to simulate these profiles as

$$
\frac{\tau_{\mathrm{L}} u *}{h}=\max \left[0.30 ; \frac{k(z-d)}{1.25^{2} h}\right]
$$

and

$$
\frac{\sigma_{\mathrm{w}}}{u *}=\left\{\begin{array}{cl}
1.25 & \text { for } z / h>1 \\
1.25-1.2 \cos \left(\frac{\pi z}{2 h}\right) & \text { for } z / h \leq 1
\end{array}\right.
$$

where $u *$ is friction velocity and $z$ is height. The decision to use these profiles was arbitrary, they do not impact the results of this study since they were consistent through all trial scenarios. 


\section{The Canopy Model}

For the model runs discussed here, the canopy and the air above it were each divided into 20 layers, with vertical leaf distribution being described by a beta function, following van den Hurk and McNaughton (1995). A simple exponential decay function was used to simulate both net radiation and irradiance within the canopy and no distinction was made between shaded and sunlit leaves. Separating sunlit and shaded leaves improves the simulation of plant $\mathrm{C}$ uptake; however, the purpose of the ICM was not to quantitatively simulate canopy photosynthesis. The use of the Ball-Berry model (discussed below) provided feedback between the environment and stomatal functioning, testing a possible role of dispersion in inducing feedback. The leaf boundary layer resistance was proportional to the square root of the ratio between leaf width $(0.02 \mathrm{~m})$ and wind speed in the layer (Jones, 1983). The height dependence of wind speed within the foliage was described by an exponential function (de Cionco, 1972) and a constant attenuation coefficient was assumed; effects of canopy architecture or leaf area index on this decay function (Meyers et al., 1998) were not included. The partitioning of the net radiation at the soil surface was imposed, with soil latent and sensible heat fluxes as boundary conditions to the canopy lowest layer, and soil heat flux $G$ as a constant fraction (0.40) of the net radiation at the soil surface (Choudhury et al., 1987). These choices were made to enable clear focus on the issue of an aerodynamic component to $r_{\mathrm{c}}$. These choices are roughly equivalent to having the model simulate a very generic canopy rather than a particular crop or ecosystem.

The leaf stomatal conductance was modeled in two ways. The first approach used the relationship by Denmead and Millar (1976) for wheat:

$$
G_{\text {st }}=0.5+0.021 R_{\text {nleaf }}
$$

where $g_{\text {st }}$ is the leaf conductance to water vapor $\left(\mathrm{mm} \mathrm{s}^{-1}\right)$ and $R_{\text {nleaf }}$ is the net radiation on the leaf $\left(\mathrm{W} \mathrm{m}{ }^{-2}\right)$. In what follows, this approach is referred to as D\&M.

The other approach used the Ball and Berry model (Ball et al. (1987), as described in Collatz et al., 1991), referred to hereafter as B\&B. This model allowed us to explore more deeply if feedback between leaf and canopy scales had some impact on the relationship between $r_{\text {res }}$ and the leaf stomatal resistance. The $\mathrm{B} \& \mathrm{~B}$ model uses the following empirical relationship to express the stomatal conductance:

$$
g_{\mathrm{sw}}=m \frac{A_{\mathrm{n}} h_{\mathrm{s}}}{c_{\mathrm{s}}}+b
$$

where $g_{\text {sw }}$ is stomatal conductance $\left(\mathrm{mol} \mathrm{m}^{-2} \mathrm{~s}^{-1}\right), A_{\mathrm{n}}$ is the rate of net $\mathrm{CO}_{2}$ uptake $\left(\mu \mathrm{mol} \mathrm{m}{ }^{-2} \mathrm{~s}^{-1}\right), h_{\mathrm{s}}$ is the relative humidity at the leaf surface, and $c_{\mathrm{s}}$ is the $\mathrm{CO}_{2}$ mole fraction at the leaf surface $\left(\mu \mathrm{mol} \mathrm{mol}{ }^{-1}\right)$. The terms $m$ and $b$ are empirical parameters, with $m=9$ and $b=0.01 \mathrm{~mol} \mathrm{~m}^{-2} \mathrm{~s}^{-1}$.

The use of the $\mathrm{B} \& \mathrm{~B}$ model necessitated the addition of a leaf assimilation model for the simulation of $A_{\mathrm{n}}$. In this context, not only does $A_{\mathrm{n}}$ contribute to determine the value of the stomatal conductance via Eq. [12] but also depends on it, since one of the factors that contributes to determine the net assimilation rate $\left(A_{\mathrm{n}}\right)$ is the $\mathrm{CO}_{2}$ mole fraction at the site of fixation $\left(c_{i}\right)$, which, in turn, depends on the magnitude of the stomatal conductance (using Ohm's analogy). This interrelationship between $A_{\mathrm{n}}$ and $g_{\mathrm{sw}}$ is of importance for the iteration procedure explained below.

The leaf assimilation model used to simulate $A_{\mathrm{n}}$ was based on the model proposed by Collatz et al. (1991) for $\mathrm{C} 3$ leaves. This model describes leaf $\mathrm{CO}_{2}$ assimilation as the minimum of three limiting rates: (i) rate limited by the efficiency of the photosynthetic enzyme system (Rubisco), (ii) rate limited by electron transport, and (iii) rate limited by the capacity of the leaf to export or utilize the products of photosynthesis. This type of approach is based on the biochemical model of leaf photosynthesis presented by Farquhar et al. (1980). Models of leaf $\mathrm{CO}_{2}$ assimilation similar to the one proposed by Collatz et al. (1991) have been used in a number of studies (Harley and Tenhunen, 1991; Sellers et al., 1996; de Pury and Farquhar, 1997; Grossman-Clarke et al., 2001), showing some diversity in the algorithms that describe the rate-limited processes, the number and type of processes assumed to limit photosynthesis, parameterization, and temperature effects included in the model. For the leaf assimilation model used in this study, not all algorithms came from Collatz et al. (1991); the electrontransport limited rate of photosynthesis followed de Pury and Farquhar (1997), as did the description of temperature effects on the $\mathrm{CO}_{2}$ compensation point and on the potential rate of electron transport.

Some of the shortcomings of Eq. [12] include breakdown at very low light, humidity, and $\mathrm{CO}_{2}$ levels (Anderson et al., 2000). In the present study, preliminary simulations using the $\mathrm{B} \& \mathrm{~B}$ model and the original Collatz et al. (1991) model produced implausible results that seemed to be related to the presence of large (simulated) temperatures within the canopy. Since some of the simulation scenarios included high temperature above the canopy, the above-mentioned modifications to the original Collatz et al. model were aimed at allowing plausible stomatal functioning at higher temperatures. In any event, the accurate simulation of leaf photosynthesis was not strictly relevant to the purpose of this study so long as the modeled stomatal functioning was comparable to a generic crop.

When the numerical simulation included the B\&B model, soil respiration became a boundary condition to the canopy's lowest layer. A constant rate of soil respiration of $0.03 \mathrm{mg} \mathrm{CO}_{2} \mathrm{~m}^{-2} \mathrm{~s}^{-1}$ (da Costa et al., 1986) was assumed and temperature effects on this rate were not considered.

\section{Iterative Procedure}

When running the model, the final profiles of latent $(\lambda E)$ and sensible $(H)$ heat fluxes within the canopy had to match two conditions: first, within each layer, fluxes between leaves and the air should be describable by the 
Ohm's Law analogy; second, the concentration profiles of temperature and water vapor within the canopy air should be defined by the flux profiles (i.e., source/sink strengths) through the dispersion matrix. To accomplish this agreement, an initial partition of net radiation into $\lambda E$ and $H$ profiles was imposed which, in turn, originated profiles of scalars within the canopy air (via the dispersion matrix). Since leaf stomatal and boundary-layer resistances were given, the Ohm's Law analogy was used in each layer to make two foliage temperature estimates, one from $H$, designated $T_{f H}$, and another from $\lambda E$, designated $T_{f E}$. If these temperatures did not coincide, the first condition was not matched and a "next foliar temperature" (and its saturation vapor value) was estimated, for each layer, based on the difference between $T_{f H}$ and $T_{f E}$, so that new profiles of $\lambda E$ and $H$ could be calculated for the next iteration, again with Ohm's law. In the next iteration, the dispersion matrix was used again and new profiles of $T_{f H}$ and $T_{f E}$ were determined. With each iteration, the difference between $T_{f H}$ and $T_{f E}$ became smaller until they coincided, meaning that the two conditions mentioned above had been met. In practice, iteration proceeded until the maximum difference between $T_{f H}$ and $T_{f E}$ was $<10^{-12}{ }^{\circ} \mathrm{C}$.

The expressions used to calculate $T_{f H}$ and the saturation vapor pressure at $T_{f E}$, designated $e_{T f E}^{*}$, in each canopy layer, were

$$
\begin{gathered}
T_{f H}=\frac{H^{\prime}}{\Lambda(z)} \frac{r_{\mathrm{bz}}}{\rho_{\mathrm{a}} c_{\mathrm{p}}}+T(z) \\
e_{T f E}^{*}=\frac{\lambda E^{\prime} \gamma}{\Lambda(z) \rho_{\mathrm{a}} c_{\mathrm{p}}}\left(\frac{1}{g_{\mathrm{st}}}+r_{\mathrm{bz}}\right)+e_{\mathrm{a}}(z)
\end{gathered}
$$

where $H^{\prime}$ and $\lambda E^{\prime}$ are sensible and latent heat flux for the canopy layer respectively, $r_{\mathrm{bz}}$ is the leaf boundary layer resistance, $T(z)$ is the air temperature at the layer level, $e_{\mathrm{a}}$ is the vapor pressure of the air at the layer level, $\Lambda(z)$ is the leaf area index for the layer, and $g_{\text {st }}$ is leaf conductance. The value $T_{f E}$ was found by inverting Tetens' equation.

When the $\mathrm{B} \& \mathrm{~B}$ model was used, the calculation of net $\mathrm{CO}_{2}$ uptake $\left(A_{\mathrm{n}}\right)$ was performed immediately after calculating latent and sensible heat fluxes. The "leaf temperature" required by the $A_{n}$ subroutine was then taken as the middle value between $T_{f H}$ and $T_{f E}$. An initial guess for stomatal conductance was needed to simulate the profile of $T_{f E}$ in the first iteration. In the first iteration, an initial profile of $A_{\mathrm{n}}$ was imposed by assuming an initial guess value for the $\mathrm{CO}_{2}$ concentration at the site of fixation $\left(c_{i}\right)$. This initial profile determined the concentration profile of $\mathrm{CO}_{2}$ within the canopy air $\left(c_{\mathrm{a}}\right)$ via the dispersion matrix. The values of $\mathrm{CO}_{2}$ concentration and relative humidity at the leaf surface $\left(c_{\mathrm{s}}\right.$ and $h_{\mathrm{s}}$ ) needed in the B\&B model were obtained, via Ohm's analogy, using outputs from both the $A_{\mathrm{n}}$ and $\lambda E$ subroutines. Stomatal conductance were estimated through the B\&B model as well as the resultant $c_{i}$ through Ohm's analogy. These values of stomatal conductance and $c_{i}$ were the ones used in the next iteration for the calculation of new $T_{f E}$ and $A_{\mathrm{n}}$ profiles. Hypothetically, the final profile of $A_{\mathrm{n}}$ should be the one capable of providing the profiles of stomatal conductance (via the $\mathrm{B} \& \mathrm{~B}$ model) and $c_{\mathrm{a}}$ (via the dispersion matrix), which will determine a profile of $c_{i}$ (via Ohm's analogy) defining the same final profile of $A_{\mathrm{n}}$ (via the leaf assimilation model). The model used in the present study was able to obtain this final profile of $A_{\mathrm{n}}$ with one reservation explained below.

The use of the $\mathrm{B} \& \mathrm{~B}$ model was not appropriate when $A_{\mathrm{n}} \rightarrow 0$ (Collatz et al., 1991). Moreover, simulated $A_{\mathrm{n}}$ was negative for some layers in the canopy when the estimated rate of gross $\mathrm{CO}_{2}$ uptake $\left(A_{\mathrm{g}}\right)$ was not larger than the estimated rate of "day respiration" $\left(R_{\mathrm{d}}\right)$ since $A_{\mathrm{n}}=A_{\mathrm{g}}-R_{\mathrm{d}}$, completely precluding the use of the $\mathrm{B} \& \mathrm{~B}$ model in these layers since negative values of stomatal conductance could then be obtained. This scenario of unrealistic estimates of stomatal conductance was overcome, in the present study, by imposing a lower limit to the stomatal conductance. In these particular cases, stomatal conductances were not determined by the value of $A_{\mathrm{n}}$ via the $\mathrm{B} \& \mathrm{~B}$ model but by an imposed lower limit. An upper limit to stomatal conductance was also imposed to avoid numerical instability. The lower and upper limits used were 0.5 and $50 \mathrm{~mm} \mathrm{~s}^{-1}$, respectively. Steady-state conditions are assumed in all simulations presented below.

\section{Scenarios for Analysis}

To examine the scaling-up problem, runs of the multilayer model were performed for a set of scenarios representing a range of environmental conditions. These scenarios were defined by systematically varying the follow variables:

1. Air temperature $(T)$ : the temperature at reference height $(2 \mathrm{~m})$ was either a hot $\left(35^{\circ} \mathrm{C}\right)$ or cool $\left(15^{\circ} \mathrm{C}\right)$ environment.

2. Air humidity $(\mathrm{RH})$ : relative humidity at reference height was set to either dry $(30 \%)$ or humid $(80 \%)$ conditions.

3. Soil evaporation $\left(\lambda E_{\mathrm{s}}\right)$ : soil evaporation was either $0 \%$ (dry soil) or $80 \%$ (wet soil) of the energy available at the soil surface $\left(R_{\mathrm{ns}}-G\right)$. The soil heat flux $(G)$ was assumed to be $40 \%$ of the net radiation reaching the soil $\left(R_{\mathrm{ns}}\right)$ for all scenarios.

4. Canopy architecture: three different profiles of leaf area density, defined by the values of the parameters $p$ and $q$ in the beta distribution function $\left(\frac{1}{\beta(p, q)} x^{p-1}(1-x)^{q-1}\right.$, where $\beta(p, q)=$ $\left.\int_{0}^{1} t^{p-1}(1-t)^{q-1} d t\right)$, were used in this study:

(a) $p ; q=2 ; 2$ : leaf area density largest in the middle of the canopy,

(b) $p ; q=4 ; 2$ : leaf area density largest in the upper layers,

(c) $p ; q=2 ; 4$ : leaf area density largest in the lower layers of the canopy.

All runs presented here used $u_{*}=0.4 \mathrm{~m} \mathrm{~s}^{-1}$. Preliminary runs, not shown here, indicated that the 
imposed $u *$ had negligible impact on the results. Net radiation $\left(R_{\mathrm{n}}\right)$ and solar irradiance $\left(I_{0}\right)$ at the top of the canopy: all runs presented here used $R_{\mathrm{n}}=700 \mathrm{~W} \mathrm{~m}^{-2}$ and $I_{0}=2000 \mu \mathrm{mol} \mathrm{m}{ }^{-2} \mathrm{~s}^{-1}$.

\section{RESULTS AND DISCUSSION}

\section{Performance of the Numerical Model}

Before addressing the scaling-up problem, we assessed whether or not the numerical model reasonably reproduced results matching our qualitative understanding of canopy micrometeorology. The goal was not to quantitatively predict either scalar profiles or canopy flux values, but to create a simulation that allows careful examination of the interaction between leaf and canopy processes as mediated by turbulent transfer.

For all scenarios, the model behaved well during the iteration procedure, converging uniformly toward a solution with less than 40 iterations. In this simulation study, no attempt was made to compare obtained profiles with experimental observations; however, every run of the model resulted in physically plausible final profiles of fluxes and scalar concentrations. Figure 1 shows the final profiles of sensible and latent heat within the canopy, air temperature and foliar temperature, and vapor pressure obtained for one particular scenario. Note that the lowest point in the flux profiles includes the soil surface flux.

\section{Canopy vs. Scaled-Up Resistance Using the D\&M Model}

The ICM output was examined in terms of $r_{\text {res }} / R_{\text {tot }}$, so that a value of one corresponds to perfect scaling from
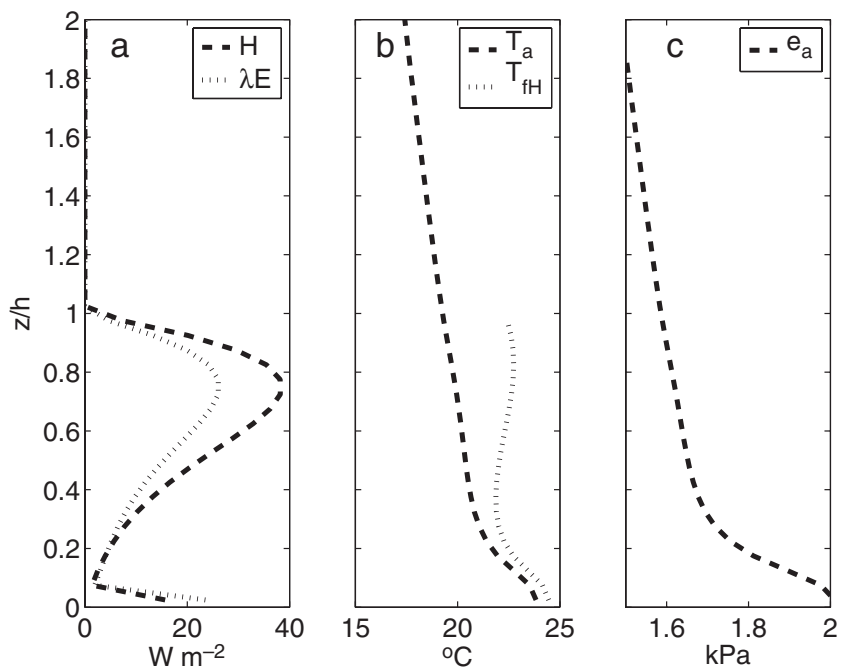

Fig. 1. Sample output from the multi-layer model for conditions defined by $R_{\mathrm{n}}=700 \mathrm{~W} \mathrm{~m}^{-2}, \Lambda=4, T=15^{\circ} \mathrm{C}, \mathrm{RH}=80 \%, \lambda E_{\mathrm{s}}=$ $60 \%, p ; q=2 ; 2$ and $u *=0.4 \mathrm{~m} \mathrm{~s}^{-1}$; leaf conductances were estimated with the D\&M algorithm; the crop height was $1 \mathrm{~m}$ and reference height was $2 \mathrm{~m}$. (a) shows the final source distribution for sensible and latent heat from the canopy ( $H_{\mathrm{p}}$ and $\lambda E_{\mathrm{p}}$, respectively) and the (fixed) soil sensible heat flux $\left(H_{\text {soil }}\right)$ and soil latent heat flux $\left(\lambda E_{\text {soil }}\right)$; (b) displays the simulated final profile of air temperature $\left(T_{a}\right)$ within and above the crop and the final profile of foliar temperature $\left(T_{f H}\right)$; (c) shows the simulated profile of water vapor pressure $\left(e_{\mathrm{a}}\right)$ for the air within and above the canopy. stomatal resistance to bulk canopy resistance. Table 1 shows the analysis for the model output under a variety of environmental conditions and for three canopy structures, using the D\&M algorithm to determine leaf-level stomatal resistance.

For leaf distributions of $p ; q=2 ; 2$ and $p ; q=4 ; 2$, the scaled-up resistance $R_{\text {tot }}$ obtained from the ICM generally agreed with the P-M residual resistance $R_{\text {resM }}$, with ratios ranging from 0.81 to 1.03 . For leaf distribution $p ; q=2 ; 4, r_{\text {res }}$ obtained with $z_{0 \mathrm{H}}=0.2 z_{0 \mathrm{M}}$ agrees better with $R_{\text {tot }}$ than $R_{\text {resM }}$, which is reasonable because the majority of the latent and sensible energy source has shifted to the lower part of the canopy. The ouput of the model is thus physically plausible, supporting the assertion that the ICM produces output appropriate for diagnosing the scaling-up problem. This result also confirms that correct estimation of $r_{\mathrm{a}}$ (through proper parameterization of $z_{0 \mathrm{H}}$ ) is important (Raupach and Finnigan, 1988).

\section{The Effect of Canopy Architecture}

The agreement between the residual resistance and the total canopy resistance depended on the choice of $z_{0 \mathrm{M}}$ or $z_{0 \mathrm{H}}$ and the leaf area distribution. Though it was suggested above that this is due to the need to correctly estimate $r_{\mathrm{a}}$, another possible explanation is that the canopy architecture, defined by the leaf area density profile, has a direct effect on the relationship between $r_{\text {res }}$ and $R_{\mathrm{tot}}$, which is independent of any indirect effect it may have on the estimation of the bulk aerodynamic resistance. To test this explanation, the model was run in

Table 1. Summary of results from different runs of the model under a variety of conditions as defined by temperature $(T)$ and relative humidity $(\mathrm{RH})$ at reference height; soil evaporation as a fraction of $R_{\mathrm{ns}}-G$. For all runs, $\Lambda=4, R_{\mathrm{n}}=700 \mathrm{~W} \mathrm{~m}^{-2}$, $u *=0.4 \mathrm{~m} \mathrm{~s}^{-1} ; R_{\text {tot }}=68 \mathrm{~s} \mathrm{~m}^{-1}$ and the $\mathrm{D \& M}$ algorithm was used to simulate leaf conductance. The ratios of residual resistance to total resistance are shown for $z_{0 \mathrm{H}}=z_{0 \mathrm{M}}\left(r_{\mathrm{resM}} /\right.$ $\left.R_{\text {tot }}\right)$ and $z_{0 \mathrm{H}}=0.2 z_{0 \mathrm{M}}\left(r_{\text {resH }} / R_{\text {tot }}\right)$. Canopy evapotranspiration from the multi-layer model $\left(\lambda E_{c}\right)$ is expressed as a ratio with respect to the energy available to the canopy $\left(A_{c}\right)$.

\begin{tabular}{|c|c|c|c|c|c|c|}
\hline$p ; q$ & $T$ & RH & Soil $\lambda E$ & $\Lambda \mathrm{E}_{\mathrm{c}} / \boldsymbol{A}_{\mathrm{c}}$ & $r_{\text {resM }} / R_{\text {tot }}$ & $r_{\text {resH }} / R_{\text {tot }}$ \\
\hline & ${ }^{\circ} \mathbf{C}$ & $\%$ & & & & \\
\hline \multirow[t]{8}{*}{$2 ; 2$} & 35 & 30 & 0 & 1.20 & 0.95 & 0.68 \\
\hline & & & 0.8 & 1.17 & 1.00 & 0.75 \\
\hline & & 80 & 0 & 0.70 & 0.88 & 1.03 \\
\hline & & & 0.8 & 0.67 & 0.98 & 1.18 \\
\hline & 15 & 30 & 0 & 0.57 & 0.91 & 0.95 \\
\hline & & & 0.8 & 0.54 & 0.98 & 1.03 \\
\hline & & 80 & 0 & 0.36 & 0.81 & 1.10 \\
\hline & & & 0.8 & 0.34 & 0.91 & 1.24 \\
\hline \multirow[t]{8}{*}{$4 ; 2$} & 35 & 30 & 0 & 1.23 & 0.90 & 0.62 \\
\hline & & & 0.8 & 1.21 & 0.93 & 0.66 \\
\hline & & 80 & 0 & 0.67 & 0.97 & 1.17 \\
\hline & & & 0.8 & 0.65 & 1.03 & 1.26 \\
\hline & 15 & 30 & 0 & 0.55 & 0.96 & 1.01 \\
\hline & & & 0.8 & 0.54 & 0.99 & 1.05 \\
\hline & & 80 & 0 & 0.33 & 0.95 & 1.31 \\
\hline & & & 0.8 & $\mathbf{0 . 3 1}$ & 1.01 & 1.40 \\
\hline \multirow[t]{8}{*}{$2 ; 4$} & 35 & 30 & 0 & 1.13 & 1.09 & 0.87 \\
\hline & & & 0.8 & 1.09 & 1.18 & 0.97 \\
\hline & & 80 & 0 & 0.75 & 0.74 & 0.83 \\
\hline & & & 0.8 & 0.70 & 0.86 & 1.00 \\
\hline & 15 & 30 & 0 & 0.60 & 0.84 & 0.85 \\
\hline & & & 0.8 & 0.56 & 0.92 & 0.96 \\
\hline & & 80 & 0 & $\mathbf{0 . 4 3}$ & 0.57 & 0.74 \\
\hline & & & 0.8 & 0.40 & 0.67 & 0.89 \\
\hline
\end{tabular}


scenarios where the air temperature was fixed at either $35^{\circ} \mathrm{C}$ or $15^{\circ} \mathrm{C}$ and the relative humidity was guessed, in each run, until the canopy transpiration provided by the multi-layer model was very close to equilibrium evaporation, making the value of $r_{\text {res }}$ independent of the bulk aerodynamic resistance; this was done for two cases: $\lambda E_{\mathrm{s}}=0$ and $\lambda E_{\mathrm{s}}=0.8\left[R_{\mathrm{ns}}-G\right]$. Results from these runs are summarized in Table 2, which shows that when $\lambda E_{\mathrm{s}}=80 \%$ of $R_{\mathrm{ns}}-G, r_{\text {res }}=R_{\text {tot }}$ irrespective of the leaf area density profile, whereas when $\lambda E_{\mathrm{s}}=0, r_{\text {res }} /$ $R_{\text {tot }}$ varied between 0.86 and 0.96 depending on $T$ and the leaf area density profile. Canopy architecture had little effect on $r_{\text {res }} / R_{\text {tot }}$, except for a small discrepancy when the soil was dry and the leaf area densest in the lower canopy.

\section{Canopy versus Scaled-Up Resistance Using the B\&B Model}

The inclusion of the B\&B model, which accounts for feedback mechanisms on the simulation of stomatal conductance, had little impact on the qualitative pattern of $r_{\text {res }} / R_{\text {tot }}$ shown by the simpler D\&M model. Table 3 shows the results of these runs using the same conditions as in Table 1 , but with $R_{\text {tot }}$ modeled according to $\mathrm{B} \& \mathrm{~B}$. As above, greater divergence between $r_{\text {res }}$ and $R_{\text {tot }}$ occurs when the bulk of the leaf mass is in the lower half of the canopy $(p ; q=2 ; 4)$. The only notable difference is that $r_{\text {res }} / R_{\text {tot }}>1$ occurs more frequently; it is not clear that any significance can be drawn from this distribution. Since these results are arguably qualitatively similar to the results using the D\&M model, they serve to further support the assertion that correct estimation of $r_{\mathrm{a}}$ is critical.

The incorporation of the B\&B model was not without problems. For the scaling-up analysis, it seemed appropriate to use quite extreme scenarios such as very hot and very dry. These conditions, however, appeared inappropriate for the $\mathrm{B} \& \mathrm{~B}$ model. The temperature response functions assumed for different parameters in the leaf assimilation model resulted in a depressing effect of high temperatures on the rate of net $\mathrm{CO}_{2}$ uptake, $A_{\mathrm{n}}$. Under these conditions then, not only did $A_{\mathrm{n}}$ diminish, but also the stomatal conductance $\left(g_{\mathrm{sw}}\right)$ calculated with the B\&B model; a feed-forward effect of

Table 2. Values of the residual resistance expressed as ratios with respect to the total canopy resistance from the multi-layer model $\left(r_{\text {res }} / R_{\text {tot }}\right)$ for different leaf area density distributions ( $p$ and $q$ ) and for cases with no soil evaporation or with soil evaporation of $80 \%$ the energy available at the soil surface. The condition for all runs is that $\lambda E_{\mathrm{c}} / A_{\mathrm{c}} \approx[\Delta /(\Delta+\gamma)]$. The scenarios were defined by $R_{\mathrm{n}}=700 \mathrm{~W} \mathrm{~m}^{-2}, \Lambda=4$, and $u_{*}=0.4 \mathrm{~m} \mathrm{~s}^{-1}$. The D\&M algorithm was used to simulate stomatal conductance, and $z_{0 \mathrm{H}} \stackrel{g}{=} z_{0 \mathrm{M}}$ was assumed.

\begin{tabular}{|c|c|c|c|}
\hline & & $r_{\text {res }} / R_{\text {tot }}$ & $r_{\text {res }} / R_{\text {tot }}$ \\
\hline $\mathbf{T}$ & $\mathbf{p} ; \mathbf{q}$ & $\Lambda E_{s}=0$ & $\Lambda E_{s}=0.8 A_{c}$ \\
\hline \multirow[t]{3}{*}{$35^{\circ} \mathrm{C}$} & $2 ; 2$ & 0.92 & 1.00 \\
\hline & $4 ; 2$ & 0.95 & 0.99 \\
\hline & $2 ; 4$ & 0.86 & 1.01 \\
\hline \multirow[t]{3}{*}{$1^{\circ} \mathrm{C}$} & $2 ; 2$ & 0.93 & 0.99 \\
\hline & $4 ; 2$ & 0.96 & 0.98 \\
\hline & $2 ; 4$ & 0.87 & 0.98 \\
\hline
\end{tabular}

Table 3. Summary of results from different runs of the multi-layer model with inclusion of the B\&B model for stomatal conductance under the same conditions used in Table 1.

\begin{tabular}{|c|c|c|c|c|c|c|c|}
\hline$p ; q$ & $\boldsymbol{T}$ & RH & soil $\lambda E$ & $R_{\text {tot }}$ & $\Lambda E_{\mathrm{c}} / A_{\mathrm{c}}$ & $r_{\text {resM }} / R_{\text {tot }}$ & $r_{\text {resH }} / R_{\text {tot }}$ \\
\hline & ${ }^{\circ} \mathbf{C}$ & $\%$ & & $\mathrm{~s} \mathrm{~m}^{-1}$ & & & \\
\hline \multirow[t]{8}{*}{$2 ; 2$} & 35 & 30 & 0 & 83.2 & 1.05 & 1.03 & 0.89 \\
\hline & & & 0.8 & 70.6 & 1.10 & 1.10 & 0.90 \\
\hline & & 80 & 0 & 42.7 & 0.81 & 0.92 & 0.94 \\
\hline & & & 0.8 & 41.1 & 0.79 & 1.05 & 1.11 \\
\hline & 15 & 30 & 0 & 89.2 & 0.46 & 0.97 & 1.09 \\
\hline & & & 0.8 & 79.1 & 0.46 & 1.08 & 1.21 \\
\hline & & 80 & 0 & 36.2 & 0.48 & 0.86 & 1.08 \\
\hline & & & 0.8 & 35.2 & 0.45 & 1.01 & 1.30 \\
\hline \multirow[t]{8}{*}{$4 ; 2$} & 35 & 30 & 0 & 105.9 & 0.95 & 0.99 & 0.92 \\
\hline & & & 0.8 & 94.1 & 1.00 & 1.00 & 0.89 \\
\hline & & 80 & 0 & 40.9 & 0.82 & 0.94 & 0.95 \\
\hline & & & 0.8 & 40.1 & 0.80 & 1.02 & 1.06 \\
\hline & 15 & 30 & 0 & 100.2 & 0.41 & 1.01 & 1.15 \\
\hline & & & 0.8 & 93.9 & 0.42 & 1.05 & 1.19 \\
\hline & & 80 & 0 & 36.7 & 0.45 & 0.97 & 1.24 \\
\hline & & & 0.8 & 36.2 & 0.44 & 1.06 & 1.37 \\
\hline \multirow[t]{8}{*}{$2 ; 4$} & 35 & 30 & 0 & 52.0 & 1.15 & 1.36 & 1.05 \\
\hline & & & 0.8 & 47.2 & 1.13 & 1.55 & 1.22 \\
\hline & & 80 & 0 & 50.9 & 0.79 & 0.84 & 0.88 \\
\hline & & & 0.8 & 46.8 & 0.76 & 1.02 & 1.12 \\
\hline & 15 & 30 & 0 & 61.9 & 0.58 & 0.98 & 1.02 \\
\hline & & & 0.8 & 55.8 & 0.55 & 1.17 & 1.23 \\
\hline & & 80 & 0 & 34.0 & 0.55 & 0.68 & 0.80 \\
\hline & & & 0.8 & 32.8 & 0.50 & 0.86 & 1.05 \\
\hline
\end{tabular}

higher temperature and lower $A_{\mathrm{n}}$ and $g_{\text {sw }}$ was created in successive iterations. In these circumstances, the multilayer model was not always able to reach a solution, or did so producing a profile of leaf conductances showing a nonrealistic shut down of stomata. The leaf assimilation model used here applies to $\mathrm{C} 3$ metabolism and temperatures close to $35^{\circ} \mathrm{C}$ may be quite extreme. Further, when the relative humidity was very low, the simulated values of stomatal conductance became very small, also creating a feed-forward effect. The range of conditions used to develop the B\&B model (Ball et al., 1987) did not include relative humidities at the leaf surface as low as those simulated in this analysis (46 vs. $30 \%$ ). The use of the B\&B model may therefore not be appropriate for relative humidity much lower than $50 \%$ at the leaf surface.

\section{Congruent Source Profiles}

Central to the derivation of $\mathrm{P}-\mathrm{M}$ is the assumption that the sources of sensible and latent heat have the same temperature (Monteith, 1981). To strictly meet this assumption requires that the profiles of sensible and latent heat fluxes be congruent, that is, constant Bowen ratio throughout the canopy layers. When this condition is not met, the meaning of a representative canopy temperature becomes dubious since it may be different for sensible and latent heat fluxes. In addition the canopy height of the hypothetical "big-leaf" surface would also not be coincident for sensible and latent heat fluxes.

In this section, we examine the relationship between $r_{\text {res }}$ and $R_{\text {tot }}$ for an ideal canopy with congruent source profiles. To do so, profiles of canopy fluxes were imposed to maintain a constant Bowen ratio; the same Bowen ratio was also imposed at the soil surface. From these imposed source profiles, the dispersion matrix 
provided profiles of air temperature and humidity. From these, profiles of foliage temperature and saturation vapor pressure were calculated, allowing for the profile of $g_{\text {st }}$ to be computed as an output by rearranging Eq. [14]. Using the profile of $g_{\text {st }}$, and the assumption that $r_{\mathrm{c}}=R_{\mathrm{tot}}$, values of $r_{\mathrm{a}}$ were computed as a residual from P-M.

This procedure was done once for one scenario for each architecture, giving three $r_{\mathrm{a}}$ values, shown in Table 4 . Also shown in Table 4 are the model output $R_{\text {tot }}$ and $r_{\text {res }} / R_{\text {tot }}$ for several scenarios using these $r_{\mathrm{a}}$ values. Under these conditions, excellent agreement between $r_{\text {res }}$ and $R_{\text {tot }}$ was obtained. Further, when these $r_{\mathrm{a}}$ values were used in the model runs shown in Table 1 (noncongruent source profiles), $r_{\text {res }} / R_{\text {tot }}$ varied between 0.96 and 1.04 for $p ; q=4 ; 2$ and 0.88 to 1.11 for $p ; q=2 ; 4$. This shows that, in the ICM, $r_{\mathrm{a}}$ is only a function of canopy architecture, as it should be, and that correct determination of this parameter essentially eliminates any difficulty in scaling-up from $g_{\text {st }}$ to $R_{\text {tot }}$ with only minor disagreement due to the violation of the congruent source profile assumption. This result further emphasizes the importance of correctly estimating $z_{0 \mathrm{H}}$ (and therefore $r_{\mathrm{a}}$ ) in applying P-M, and shows that both parameterizations used in this study are limited in applicability to certain specific conditions.

\section{SUMMARY}

The large discrepancies between the inverse of the sum of leaf conductances $\left(R_{\mathrm{tot}}\right)$ and the bulk canopy resistance $\left(r_{\text {res }}\right)$ sometimes reported in the literature may be due to incorrect estimation of $r_{\mathrm{a}}$, which in turn depends on the choice of $z_{0 \mathrm{H}}$ parameterization. If this choice does not produce an accurate estimation of the aerodynamic temperature as the representative temperature of the transpiring elements, the fundamental assumption of $\mathrm{P}-\mathrm{M}$ is violated.

Some caution is advisable, then, when large discrepancies between $r_{\text {res }}$ and $R_{\text {tot }}$ are reported. It could be the case that the models involved in the estimation of these resistances (the Penman-Monteith equation for $r_{\text {res }}$ and some other model for the estimation of $\left.R_{\text {tot }}\right)$ are not able to adequately describe the scenarios under consideration, due to, for example, noncongruent source profiles. A practical inconsistency between $r_{\text {res }}$ and $R_{\text {tot }}$ should not automatically imply that $r_{\mathrm{c}}$ is not a strict physiological parameter. In some cases, it could also mean that either $R_{\text {tot }}$ or the profile of leaf conductances is not being modeled or measured accurately. Moreover, it could be that the Penman-Monteith equation is being used for a scenario for which it is not appropriate, such as when the canopy is partly wet.

\section{CONCLUSIONS}

The creation of the ideal canopy model was successful. The model was able to produce unique, reasonable profiles of fluxes and scalar concentrations that satisfied both the leaf and the dispersion model. Problems encountered when the $\mathrm{B} \& \mathrm{~B}$ model was used to simulate leaf conductance under high temperature and low humidity conditions are inherent to the $\mathrm{B} \& \mathrm{~B}$ model and its leaf assimilation subroutine. It was not a goal of this study to assess the B\&B model but the overall behavior suggests that its applicability within a model solving iteratively for a wide range of conditions, including a sparse canopy, may be problematic.

In the present study simulated values of $r_{\text {res }}$ and $R_{\text {tot }}$ were generally close irrespective of the inclusion of feedback mechanisms when estimating leaf conductances. Moreover, hypothetical canopies with congruent source profiles always had $r_{\text {res }}=R_{\text {tot }}$. The results suggest that there could be another explanation to the large discrepancies between $r_{\text {res }}$ and $R_{\text {tot }}$ sometimes reported in the literature. To pursue this issue, a distinction has to be made between $r_{\text {res }}$ and $r_{\mathrm{c}}$. The bulk canopy resistance used in the P-M equation is the canopy resistance. The problem with this resistance is that it cannot be measured directly and independently; it can only be estimated by rearranging the P-M equation. It is then more properly termed the residual resistance.

The Penman-Monteith equation, as formulated in Eq. [1], implies that (i) fluxes between source-sink height and reference height have constant values, meaning that no flux convergence/divergence exists between these points; (ii) the energy available to the system equals the sum of sensible and latent heat fluxes; (iii) the aerodynamic resistance between these points is properly described by a bulk value $r_{\mathrm{a}}$; (iv) the same bulk aero-

Table 4. Summary of results using imposed energy flux profiles with constant Bowen ratio profile. Column definitions as in previous tables. For all runs, $\Lambda=4$ and $R_{n}=700 \mathrm{~W} \mathrm{~m}$; the D\&M algorithm was used to simulate leaf conductance. Canopy evapotranspiration from the multi-layer model $\left(\lambda E_{c}\right)$ is expressed as a ratio with respect to the energy available to the canopy $\left(A_{c}\right)$.

\begin{tabular}{|c|c|c|c|c|c|c|c|c|}
\hline \multirow[b]{3}{*}{$T$} & \multirow[b]{3}{*}{ RH } & \multirow[b]{3}{*}{$\Lambda E_{c} / A_{c}$} & \multirow{2}{*}{\multicolumn{2}{|c|}{$\begin{array}{c}p ; q=2 ; 2 \\
r_{\mathrm{a}}=15.6 \mathrm{~s} \mathrm{~m}^{-1}\end{array}$}} & \multirow{2}{*}{\multicolumn{2}{|c|}{$\begin{array}{c}p ; q=4 ; 2 \\
r_{\mathrm{a}}=12.8 \mathrm{~s} \mathrm{~m}^{-1}\end{array}$}} & \multirow{2}{*}{\multicolumn{2}{|c|}{$\begin{array}{c}p ; q=2 ; 4 \\
r_{\mathrm{a}}=24.4 \mathrm{~s} \mathrm{~m}^{-1}\end{array}$}} \\
\hline & & & & & & & & \\
\hline & & & $R_{\text {tot }}$ & $r_{\text {res }} / R_{\text {tot }}$ & $R_{\text {tot }}$ & $r_{\text {res }} / R_{\text {tot }}$ & $R_{\text {tot }}$ & $r_{\text {res }} / R_{\text {tot }}$ \\
\hline${ }^{\circ} \mathbf{C}$ & & & $\mathrm{s} \mathrm{m}^{-1}$ & & $\mathrm{~s} \mathrm{~m}^{-1}$ & & $\mathrm{~s} \mathrm{~m}^{-1}$ & \\
\hline \multirow[t]{5}{*}{35} & 0.3 & 0.30 & 546 & 1.00 & 510 & 0.99 & 674 & 1.00 \\
\hline & & 0.82 & 132 & 1.00 & 132 & 1.00 & 133 & 1.00 \\
\hline & & 1.10 & 76 & 1.00 & 80 & 1.01 & 63 & 1.02 \\
\hline & 0.7 & 0.30 & 336 & 1.01 & 300 & 1.00 & 460 & 1.01 \\
\hline & & 0.82 & 56 & 1.01 & 56 & 1.01 & 57 & 1.02 \\
\hline \multirow[t]{4}{*}{15} & 0.3 & 0.30 & 167 & 1.00 & 155 & 0.99 & 210 & 0.99 \\
\hline & & 0.62 & 55 & 1.00 & 55 & 1.00 & 58 & 0.99 \\
\hline & 0.7 & 0.30 & 103 & 1.01 & 92 & 1.00 & 145 & 1.00 \\
\hline & & 0.62 & 25 & 1.00 & 24 & 1.00 & 27 & 1.00 \\
\hline
\end{tabular}


dynamic resistance is valid for both sensible and latent heat, in other words $r_{\mathrm{aH}}=r_{\mathrm{av}}$; (v) the aerodynamic temperature is a good estimate of the actual mean conditions on the surfaces of the transpiring elements; and (vi) the scenario under analysis corresponds to one of the intended cases for its use. Violation of any of these requirements in a specific situation would mean that the use of the Penman-Monteith equation is not appropriate or that not all the variables within the equation are being estimated or measured correctly. Only when all of these requirements are satisfied may one have confidence that $r_{\text {res }}$ is a good estimate of $r_{\mathrm{c}}$.

Failure to satisfy some of the requirements mentioned above, on the other hand, could cause a discrepancy between $r_{\text {res }}$ and $R_{\text {tot }}$ that should not be interpreted as a weak physiological significance of the bulk canopy resistance since, in this case, the problem could rely on the discrepancy between $r_{\text {res }}$ and $r_{\mathrm{c}}$. The $r_{\text {res }}$ that is "measured" in practice is just a residual term so that its value is merely the one that forces some formulation of the Penman-Monteith equation to work in a specific situation. In this context, a large discrepancy between $r_{\text {res }}$ and $R_{\text {tot }}$ could also be evidence of a situation departing markedly from the P-M model just described.

The results of this study support $r_{\mathrm{c}}$ being a physiological parameter that integrates all stomatal resistances within the canopy, and $r_{\mathrm{a}}$ being strictly aerodynamic. The correct estimation of $r_{\mathrm{a}}$, even if $r_{\mathrm{aH}}=r_{\mathrm{aV}}$ holds for the situation under analysis, is particularly important since it contributes to the determination of the value of the aerodynamic temperature, surrogate for the actual mean temperature of the transpiring elements. The determination of $z_{0 \mathrm{H}}$ and the method used to correct for nonneutral atmospheric conditions are important factors in the estimation of $r_{\mathrm{a}}$; none of these procedures are without problems.

\section{REFERENCES}

Alves, I., A. Perrier, and L. Pereira. 1998. Aerodynamic and surface resistances of complete cover crops: How good is the 'big leaf'? Trans. ASAE 41:345-351.

Anderson, M., J. Norman, T. Meyers, and G. Diak. 2000. An analytical model for estimating canopy transpiration and carbon assimilation fluxes based on canopy light-use efficiency. Agric. For. Meteorol. 101:265-289.

Ball, J., I. Woodrow, and J. Berry. 1987. A model predicting stomatal conductance and its contributions to the control of photosynthesis under different environmental conditions. p. 221-224. In J. Biggins (ed.) Prog. Photosynth. Res. Proc. Int. Congr. 7, Providence. 10-15 Aug. 1986. Kluwer Academic Publishers, Dordrecht, the Netherlands.

Choudhury, B., S. Idso, and R. Reginato. 1987. Analysis of an empirical model for soil heat flux under a growing wheat crop for estimating evaporation by an infrared-temperature based energy balance equation. Agric. For. Meteorol. 39:283-297.

Collatz, G., J. Ball, C. Grivet, and J. Berry. 1991. Physiological and environmental regulation of stomatal conductance, photosynthesis and transpiration: A model that includes a laminar boundary layer. Agric. For. Meteorol. 54:107-136.

da Costa, J., N. Rosenberg, and S. Verma. 1986. Respiratory release of $\mathrm{CO}_{2}$ in alfalfa and soybean under field conditions. Agric. For. Meteorol. 37:143-157.

de Cionco, R. 1972. A wind-profile index for canopy flow. BoundaryLayer Meteorol. 3:255-263.

de Pury, D., and G. Farquhar. 1997. Simple scaling of photosynthesis from leaves to canopies without the errors of big-leaf models. Plant Cell Environ. 20:537-557.

Denmead, O., and B. Millar. 1976. Field studies of the conductance of wheat leaves and transpiration. Agron. J. 68:307-311.

Farquhar, G., S. van Caemmerer, and J. Berry. 1980. A biochemical model of photosynthetic $\mathrm{CO}_{2}$ fixation in leaves of $\mathrm{C} 3$ species. Planta 149:78-90.

Grossman-Clarke, S., P. Pinter, Jr., T. Kartschall, B. Kimball, D. Hunsaker, G. Wall, R. Garcia, and R. LaMorte. 2001. Modelling a spring wheat crop under elevated $\mathrm{CO}_{2}$ and drought. New Phytol. 150:315-335.

Harley, P., and J. Tenhunen. 1991. Modeling the photosynthetic response of c leaves to environmental factors. p. 17-39. In Modeling photosynthesis - from biochemistry to canopy. CSSA, Madison, WI.

Jones, H. 1983. Plants and microclimate. Cambridge Univ. Press, Cambridge, UK.

Meyers, T., P. Finkelstein, J. Clarke, T. Ellestad, and P. Sims. 1998 A multi-layer model for inferring dry deposition using standard meteorological measurements. J. Geophys. Res. 103:22645-22661.

Monteith, J. 1965. Evaporation and environment. p. 205-234. In The state and movement of water in living organisms. Vol. 19. Symp. Soc. Exp. Biol. Cambridge Univ. Press, Cambridge, UK.

Monteith, J. 1981. Evaporation and surface temperature. Q. J. R. Meteorol. Soc. 107:1-27.

Paw U, K., and T. Meyers. 1989. Investigations with a higher-order canopy turbulence model into mean source-sink levels and bulk canopy resistances. Agric. For. Meteorol. 47:259-271

Raupach, M. 1987. A Lagrangian analysis of scalar transfer in vegetation canopies. Q. J. R. Meteorol. Soc. 113:107-120.

Raupach, M. 1995. Vegetation-atmosphere interaction and surface conductance at leaf, canopy and regional scales. Agric. For. Meteorol. 73:151-179.

Raupach, M., and J. Finnigan. 1988. Single-layer models of evaporation from plant canopies are incorrect but useful, whereas multi-layer models are correct but useless. Aust. J. Plant Physiol. 15:705-716.

Rochette, P., E. Pattey, R. Desjardin, L. Dwyer, D. Stewart, and P. Dubé. 1991. Estimation of maize (Zea mays L.) canopy conductance by scaling up leaf stomatal conductance. Agric. For. Meteorol. 54:241-261.

Sellers, P., E. Randall, G. Collatz, J. Berry, C. Field, D. Dazlich, C. Zhang, G. Collelo, and L. Bounoua. 1996. A revised land surface parameterization (SiB2) for atmospheric GCMs: I. Model formulation. J. Clim. 9:676-705.

Shuttleworth, W. 1976. A one-dimensional theoretical description of the vegetation-atmosphere interaction. Boundary-Layer Meteorol. 10:273-302.

Taylor, G. 1921. Diffusion by continuous movements. Proc. Lond. Math. Soc., Ser. 2 20:196-211.

Thom, A. 1975. Momentum, mass and heat exchange of plant communities. In J. Monteith (ed.) Vegetation and the atmosphere. Academic Press, New York.

van den Hurk, B., and K. McNaughton. 1995. Implementation of nearfield dispersion in a simple two-layer surface resistance model J. Hydrol. 166:293-311.

Warland, J., and G. Thurtell. 2000. A Lagrangian solution to the relationship between a distributed source and concentration profile. Boundary-Layer Meteorol. 96:453-471. 